\title{
Maximal Models of Assertion Graph in GSTE
}

\author{
Guowu Yang ${ }^{1}$, Jin Yang $^{2}$, Xiaoyu Song ${ }^{3}$, and Fei Xie ${ }^{1}$ \\ 1 Dept. of Computer Science, Portland State University, Portland, OR 97207, USA \\ 2 Intel Corporation, Strategic CAD Research Labs, Hillsboro, OR 97124, USA \\ 3 Dept. of Electrical and Computer Engineering, \\ Portland State University, Portland, OR 97207, USA
}

\begin{abstract}
Generalized symbolic trajectory evaluation (GSTE) is an extension of symbolic trajectory evaluation (STE). In GSTE, assertion graphs are used to specify properties in a special form of regular automata with antecedent and consequent pairs. This paper presents a new model characterization, called maximal models, for an assertion graph with important properties. Besides their own theoretical significance, maximal models are used to show the implication of two assertion graphs in GSTE. We show that, contrary to the general belief, an assertion graph may have more than one maximal model. We present a provable algorithm to find all maximal models of a linear assertion graph. We devise an algorithm for finding a maximal model for an arbitrary assertion graph.
\end{abstract}

\section{Introduction}

Generalized symbolic trajectory evaluation (GSTE) [1, 2] is an extension of symbolic trajectory evaluation (STE) [5]. STE can handle large, industrial design and has been actively used in HP, IBM, and Motorola [9, 10, 11, 12. The STE theory consists of a simple specification language, a simulation-based model checking algorithm, and a mapping of the algorithm to a coarse abstract domain. The specification language of STE has the limited expressiveness where only properties over finite time intervals are allowed. GSTE was originally developed at Intel and has successfully demonstrated its powerful capacity in formal verification of digital systems [1, 2, 3, 4, 13, 14.

In GSTE, all Omega-regular properties can be expressed and verified with the same space efficiency and comparable time efficiency. Assertion graphs are introduced in GSTE as an extension of STE's specification language. Assertion graphs are the specification language in GSTE based on a special form of regular automata with assertion letters (antecedent and consequent pairs) 22. GSTE specifications are expressed in the form of assertion graphs.

Many RTL designs are rather complicated, primarily because they model complex functional behavior while accommodating tight performance constraints. If we have already proved an assertion graph $G_{1}$ against the RTL, a desirable usage is to use $G_{1}$ to prove (imply) another assertion graph $G_{2}$. Having such an implication mechanism would enable us to achieve higher level abstractions and 
pursue assume-guarantee prove strategies. There is some work on the implication [3, 4]. In this paper, we present a new concept: maximal model of an assertion graph. Maximal models are used to show the implication of two assertion graphs in GSTE.

This paper is organized as follows. In Section 2, we introduce the basic definitions in GSTE. In Section 3, we introduce some concepts, such as sub-model, maximal model and related properties. In Section 4, we present a provable algorithm to find all maximal models for a linear assertion graph. The application of maximal models in the model-based implication is discussed. In Section 5, we present an algorithm to find a maximal model of an arbitrary assertion graph. We give a condition to determine if a model is a maximal model. In Section 6, we conclude the paper.

\section{Preliminaries}

We introduce some basic definitions on GSTE [1, 2]. We assume a non-empty set of finite states, denoted by $S$. A relation $T \subseteq S \times S$ is a transition relation if $\forall s \in S, \exists s^{\prime} \in S,\left(s, s^{\prime}\right) \in T$, where $S$ is a non-empty set of finite states. The model $M$ induced by the transition relation $T$ is the pair (pre,post) where: (1) the preimage transformer pre : $2^{S} \rightarrow 2^{S}$ is defined as: $\operatorname{pre}(Q)=\left\{s \mid s^{\prime} \in Q,\left(s, s^{\prime}\right) \in T\right\}$ for all $Q \in 2^{S}$; and (2) the post-image transformer post : $2^{S} \rightarrow 2^{S}$ is defined as: $\operatorname{post}(Q)=\left\{s^{\prime} \mid s \in Q,\left(s, s^{\prime}\right) \in T\right\}$ for all $Q \in 2^{S}$.

In fact, a model $M=$ (pre,post) is a directed graph $M=(S, T)$. We use pre and post to represent two functions based on $M$. Note that $\operatorname{pre}(s)=\operatorname{pre}(s)$, $\operatorname{post}(s)=\operatorname{post}(s)$, for all $s \in S$. If for all $s \in S, \operatorname{post}(s)$ is defined and nonempty, then $M$ is well-defined. Namely, if we first define post $: S \rightarrow 2^{S}-\{\emptyset\}$, where $\emptyset$ is an empty set, then a transition relation $T$ can be defined as $T=\left\{\left(s, s^{\prime}\right) \mid s \in\right.$ $\left.S, s^{\prime} \in \operatorname{post}(s)\right\}$. A trace in $M=($ pre, post) is a state sequence such that $\sigma[i+1] \in$ $\operatorname{post}(\sigma[i])$, for all $1 \leq i<|\sigma|$, i.e., $(\sigma[i], \sigma[i+1]) \in T$.

An assertion graph is a quintuple $G=\left(V, v_{0}, E\right.$, ant, cons $)$ where $V$ is a finite set of vertices, $v_{0}$ is the initial vertex, $E \subseteq V \times V$ is a set of edges, satisfying $\forall u \in V, \exists v \in V$, such that $(u, v) \in E$, ant is a mapping: $E \rightarrow 2^{S}$, cons is a mapping: $E \rightarrow 2^{S}$. Let $G=\left(V, v_{0}, E\right.$, ant, cons $)$ be an assertion graph, and let $M=$ (pre, post) be a model. We define an edge labeling $\gamma$ as $: E \rightarrow 2^{S}$ where $\gamma$ is either ant or cons. A trace in $M$ satisfies a path $\rho$ of the same length under $\gamma$, denoted by $(M, \sigma) \models_{\gamma}(G, \sigma)$, iff $\sigma[i] \in \gamma(\rho[i]), 1 \leq i \leq|\sigma|$. A trace satisfies a path, denoted by $(M, \sigma) \models(G, \rho)$, iff $[(M, \sigma) \models$ ant $(G, \rho)] \Rightarrow\left[(M, \sigma) \models_{\text {cons }}(G, \rho)\right]$.

Let $\operatorname{ban}(e)=\operatorname{ant}(e)-\operatorname{cons}(e)$. For a trace $\sigma$ and a path $\rho$ with length $k$, if the trace with the first $k-1$ elements of $\sigma$ ant satisfies the path with the first $k-1$ elements of $\rho$, then $(M, \sigma) \models(G, \rho)$ if and only if $\sigma[k]$ is not in $\operatorname{ban}(\rho[k])$. A model $M$ strongly satisfies an assertion graph $G$, denoted by $M \models G$ iff $(M, \sigma) \models(G, \rho)$ for all finite initial path $\rho$ in $G$ and all finite trace $\sigma$ in $M$ of the same length. Given two assertion graphs $G_{1}=\left(V, v_{0}, E_{1}\right.$, ant $_{1}$, cons $\left._{1}\right)$ and $G_{2}=$ $\left(U, u_{0}, E_{2}\right.$, ant $_{2}$, cons $\left._{2}\right), G_{1}$ model-based implies $G_{2}$, denoted by $G_{1} \Rightarrow_{\text {model }} G_{2}$ (we simply denoted by $G_{1} \Rightarrow G_{2}$ ), iff $\forall M, M \models G_{1} \Rightarrow M \models G_{2}$. 
We impose two restrictions on an assertion graph:

Assumption 1: for all initial edge $e$ (i.e., $\left.\operatorname{start}(e)=v_{0}\right)$, $\operatorname{ban}(e)=\emptyset$, i.e., $\operatorname{ant}(e)=\operatorname{cons}(e)$;

Assumption 2: for all $e, \operatorname{ant}(e) \neq \emptyset$.

First, if there is an initial edge $e$ such that $\operatorname{ban}(e) \neq \emptyset$, then the one-length trace $s(s \in \operatorname{ban}(e))$ does not satisfy the path $e$, which means no model satisfies this an assertion graph. Second, if $\operatorname{ant}(e)=\emptyset$ for some edge $e$, then all successor edges of $e$ do not affect models.

\section{Maximal Model}

In this section, we define some concepts such as submodel and maximal model, and give some properties on them.

\section{Definition 1 (Submodel or Contained)}

i) Given two models: $M_{1}=\left(S_{1}, T_{1}\right), M_{2}=\left(S_{2}, T_{2}\right)$, where $S_{1} \subseteq S, S_{2} \subseteq S$, if $S_{1} \subseteq S_{2}$, and $T_{1} \subseteq T_{2}$, then $M_{1}$ is called a submodel of $M_{2}$, or $M_{1}$ is contained by $M_{2}$, denoted by $M_{1} \leq M_{2}$.

ii) If $S_{1} \subseteq S_{2}$, and $T_{1} \subset T_{2}$, then $M_{1}$ is called a proper submodel of $M_{2}$, or $M_{1}$ is properly contained by $M_{2}$, denoted by $M_{1}<M_{2}$.

Theorem 1. If $M_{1} \leq M_{2}$, and $M_{2} \models G$, then $M_{1} \models G$.

Definition 2 (Maximal Model). A Maximal-Model of an assertion graph $G$ is a model $M=(S, T) \models G$ and we can not find another model $M_{1}=\left(S, T_{1}\right) \models G$ such that $T \subset T_{1}$. Denoting $M_{-} \max _{-} G=\{M \mid M$ is a maximal-model of $G\}$.

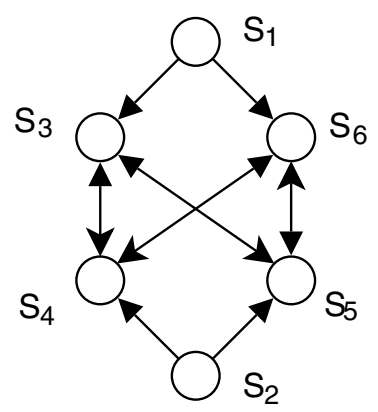

Fig. 1. A model

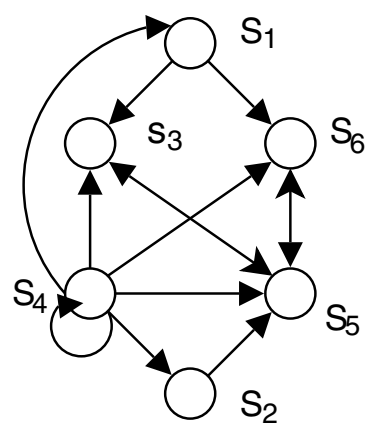

Fig. 1(b). A model

Theorem 2. $\left(G_{1} \Rightarrow G_{2}\right) \Leftrightarrow\left(\forall M, M \in M \_\max _{-} G_{1} \Rightarrow M \models G_{2}\right)$.

Example 1. Models in Fig.1 and 1(b) are both the maximal models of $G$ in Fig.2.

Theorem 3. For any given model $M=(S, T)$, there exists an assertion graph $G$ such that $M$ is the unique maximal model of $G$. 


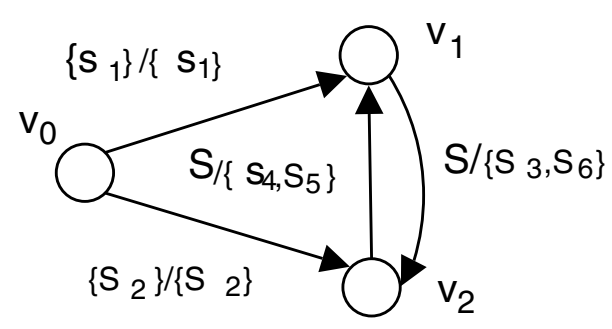

Fig. 2. An assertion graph

The maximal-model of an assertion graph $G$ is usually not unique (see Example 1). Theorem 3 illustrates that only one maximal model of $G_{1}$ satisfying $G_{2}$ is not enough to derive $G_{1} \Rightarrow G_{2}$ if $G_{1}$ has at least two maximal models. From Example 2 in Section 4, we can see how to use the maximal models to determine $G_{1} \Rightarrow G_{2}$.

\section{$4 \quad$ Finding all Maximal Models}

In this section, we consider the problem of finding all maximal models of a linear assertion graph $G$. From Theorem 2, if we find all maximal models of an assertion graph $G_{1}$, then we can determine that $G_{1}$ model-based implicates $G_{2}$. We present the following algorithm: Computing All Maximal Models (CAMM) which can find all maximal models of $G$.

Definition 3 (Linear assertion graph). $G=\left(V, v_{0}, E\right.$, ant, cons $)$ : Every edge has one and only one successor edge. In the following, without special announcement, if an assertion graph $G$ is a linear assertion graph, we always assume that $|E|=m, e[m]=\left(v_{m-1}, v_{t}\right), 0 \leq t \leq m-1, e[m+1]=e[t+1]$, namely, from $m+1$, edges have a periodicity $\tau=m-t$ (Fig.3).

\section{Algorithm: CAMM (G)}

1. $\forall s \in S, P_{1}(s)=S, Q_{1}=S, A_{1}=\operatorname{ant}\left(e_{1}\right) \cap Q_{1}=\operatorname{ant}\left(e_{1}\right)$;

2. for $i$ from 1 to $t-1$ do

3. If $s \in A_{i}$, then $P P_{i+1}(s)=P_{i}(s)-\operatorname{ban}\left(e_{i+1}\right)$;

4. else, $P P_{i+1}(s)=P_{i}(s)$;

5. $Q Q_{i+1}=\cup_{s \in A_{i}} P P_{i+1}(s)$;

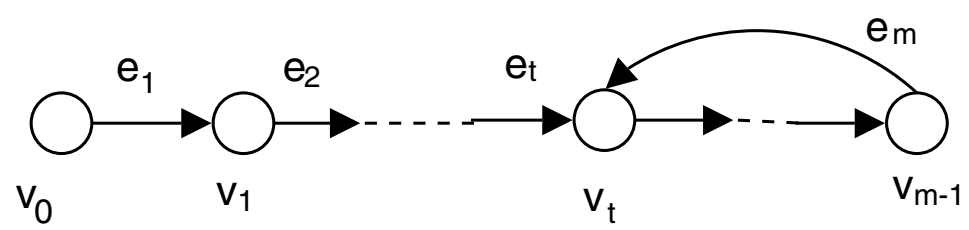

Fig. 3. Linear assertion graph 
6. $A A_{i+1}=\operatorname{ant}\left(e_{i+1}\right) \cap Q Q_{i+1}$;

7. $C_{i+1}=$ subset $A A_{i+1}$;

8. If $s \in A A_{i}$, then $P_{i+1}(s)=P_{i}(s)-\operatorname{ban}\left(e_{i+1}\right)-C_{i+1}$;

9. $Q_{i+1}=\cup_{s \in A_{i}} P_{i+1}(s)$;

10. $A_{i+1}=\operatorname{ant}\left(e_{i+1}\right) \cap Q_{i+1}$;

11. End for;

12. $k=t$;

13. for $j$ from 1 to $\tau-1$ do

14. $i=k-1+j$;

15. If $s \in A_{i}$, then $P P_{i+1}(s)=P_{i}(s)-\operatorname{ban}\left(e_{i+1}\right)$;

16. else, $P P_{i+1}(s)=P_{i}(s)$;

17. $Q Q_{i+1}=\cup_{s \in A_{i}} P P_{i+1}(s)$;

18. $A A_{i+1}=\operatorname{ant}\left(e_{i+1}\right) \cap Q Q_{i+1}$;

19. $C_{i+1}=\operatorname{subset} A A_{i+1}$;

20. If $s \in A A_{i}$, then $P_{i+1}(s)=P_{i}(s)-\operatorname{ban}\left(e_{i+1}\right)-C_{i+1}$;

21. $Q_{i+1}=\cup_{s \in A_{i}} P_{i+1}(s)$;

22. $A_{i+1}=\operatorname{ant}\left(e_{i+1}\right) \cap Q_{i+1}$;

23. End for;

24. If $P_{k+\tau}(s)=P_{k}(s)$ for all $s \in S$ and $Q_{k+\tau}=Q_{k}$, goto 26;

25 . Else $k=k+\tau$, goto 13 ;

26. Return $P^{*}(s)=P_{k}(s)$ for all $s \in S$.

Starting with a trivial model $M_{0}: \operatorname{post}(s)=S$, for every state $s$, the algorithm reduces the set of reachable states $P_{i}(s)$ for each state $s$ edge by edge until it finds a fix-point $P^{*}(s)$. Let $A_{1}=\operatorname{ant}\left(e_{1}\right)$ be the set of initial states which are constrained by the second edge. For $s \in A_{1}$, the set of reachable states $P P_{2}(s)$ from $s$ is limited by the second edge $e_{2}$. Let $Q Q_{1}$ be the union of $P P_{2}(s)$ for $s \in A_{1}$. Let $A A_{2}$ be the set of the states that are limited by the 3rd edge. The set of states $\operatorname{ban}\left(e_{2}\right)$ are removed from $P_{1}(s)$. Let $C_{2}$ contain the states that are forced to reduce from $P_{2} P_{2}(s) . C_{2}$ is a subset of $A A_{2}$. Let $P_{2}(s)$ be the final set of reachable states of $\mathrm{s}$ after limitation by the 2 nd edge. Let $A_{2}$ be the final set of states to be limited by the 3rd edge. Repeating the same process, we continue the computation of $P_{i}(s)$ until no states will be removed from $P_{i}(s)$. As a result, $P_{i}(s)$ monotonically decreases to a fix-point $P^{*}(s)$. We obtain a model $M$ such that $\operatorname{post}(s)=P^{*}(s)$. CAMM is devised to attain the models including all the maximal models. Example 4.1 shows the process.

Let $M\left(\right.$ subset $A A_{2}$, subset $A A_{3}, \ldots$, subset $\left.A A_{h}\right)$ be an output model produced by algorithm CAMM, where $C_{j}=\emptyset, j>h$.

Theorem 4. For any given maximal model $M$ of $G$, there is a model $M\left(\right.$ subset $A A_{2}$, subset $A A_{3}, \ldots$, subset $\left.A A_{l}\right)=M$.

Example 2. Given two assertion graphs $G_{1}$ and $G_{2}$, with a same directed graph: two vertices: $v_{0}, v_{1}$, and two edges: $e_{1}=\left(v_{0}, v_{1}\right), e_{2}=\left(v_{1}, v_{1}\right)$. 
$G_{1}:$ ant $_{2}\left(e_{1}\right)=$ cons $_{2}\left(e_{1}\right)=\{2\}$, ant $_{2}\left(e_{2}\right)=\{2,3,4,5\}$, cons $_{2}\left(e_{2}\right)=\{2,4,5\}$, $\operatorname{ban}_{2}\left(e_{2}\right)=\{3\}$,

$G_{2}: \operatorname{ant}_{1}\left(e_{1}\right)=\operatorname{cons}_{1}\left(e_{1}\right)=\{2\}, \operatorname{ant}_{1}\left(e_{2}\right)=\{3,4,5,6,7\}, \operatorname{cons}_{1}\left(e_{2}\right)=$ $\{5,6\}, \operatorname{ban}_{1}\left(e_{2}\right)=\{3,4,7\}$.

Using CAMM, $(S=\{1,2,3,4,5,6,7\})$, we have four models:

$M_{1}: P(2)=P(5)=P(6)=\{1,2,5,6\}, P(1)=P(3)=P(4)=P(7)=S$.

$M_{2}: P(2)=P(5)=\{1,2,5\}, P(1)=P(3)=P(4)=P(6)=P(7)=S$.

$M_{3}: P(2)=P(6)=\{1,2,6\}, P(1)=P(3)=P(4)=P(5)=P(7)=S$.

$M_{4}: P(2)=\{1,2\}, P(1)=P(3)=P(4)=P(5)=P(6)=P(7)=S$.

Using SMC in [1,2], we know that $M_{i} \models G_{2}$ for $i=1,2,3,4$. Therefore, $G_{1} \Rightarrow G_{2}$. In fact, these four models are maximal models of $G_{1}$ according to Theorem 8. For the model-based implication, we know in [15] if two assertion graphs $G_{1}$ and $G_{2}$ have the same graph structure and $\operatorname{ant}_{2}(e) \subseteq$ $\left.\operatorname{ant}_{1}(e)\right) \wedge\left(\operatorname{cons}_{1}(e) \cap \operatorname{ant}_{2}(e) \subseteq \operatorname{cons}_{2}(e)\right)$, for all $e \in E$, namely, $\left(\operatorname{ant}_{1}(e) \supseteq\right.$ $\left.\operatorname{ant}_{2}(e)\right) \wedge\left(\operatorname{ban}_{1}(e) \supseteq \operatorname{ban}_{2}(e)\right)$, for all $e \in E$, then we have $G_{1} \Rightarrow G_{2}$. In example 2 , ant ${ }_{1}(e) \supseteq \operatorname{ant}_{2}(e)$ is not true, but $G_{1} \Rightarrow G_{2}$, which means this sufficient condition for model-based implication is not necessary. For linear assertion graphs, [15] gave the sufficient and necessary conditions for language-based implication. But for model-based implication, the problem is more complicated. Example 2 shows that these conditions are not either sufficient or necessary for model-based implication.

\section{Finding a Maximal Model of an Arbitrary Assertion Graph}

Let $\operatorname{start}(e)$ and $e n d(e)$ denote the start and end vertices of a directed edge $e$, respectively. Let $\operatorname{start}(v)$ and $\operatorname{end}(v)$ denote the directed edges in an assertion graph $G$ with the starting vertex $v$ and the ending vertex $v$, respectively. We define the following sets for an assertion graph $G=\left(V, v_{0}, E\right.$, ant, cons $)$ :

$$
\begin{aligned}
& V_{0}=\left\{v_{0}\right\}, E_{1}=\left\{e \mid \operatorname{start}(e)=v_{0}\right\}, \\
& V_{1}=\left\{v \mid e \in E_{1}, \operatorname{end}(e)=v\right\}, \\
& E_{i}=\left\{e \mid \operatorname{start}(e) \in V_{i-1}\right\}, V_{i}=\left\{v \mid e \in E_{i}, \operatorname{end}(e)=v\right\}, \text { for } i=2,3, \ldots
\end{aligned}
$$

Lemma 1. There exist $t$ and $\tau>0$ such that $V_{t}=V_{t+\tau}$.

Let $t$ and $\tau$ be the minimum numbers satisfying $V_{t}=V_{t+\tau}$. We present an algorithm, Computing Satisfied Model (CSM), to find a maximal model of an arbitrary assertion graph. In the algorithm, we compute the set of reachable states $P_{i}(s)$ from state $s$ after the restriction of the ith step for all $s \in S$.

The basic idea of the algorithm CSM is described as follows. We initialize $P_{1}(s)=$ post_$M(s)$, for all $s \in S$. Initially, the set of reachable states from any state $s$ is the post function of $s$ in an input model $M$. Along the path of the assertion graph from the initial edges $E_{1}$, we reduce the set of the states reachable from s. $A_{1}(v)=\cup_{e \in E_{1}}$ ant $(e), v \in V_{1}$, is the initial states which will be constrained by the edges $E_{2}$. For $s \in A_{1}(v)$, the reachable states $P_{2}(s)$ from 
$s$ will be limited by the edges of $\operatorname{start}(v)$. The states of $\operatorname{ban}(v)$ will be removed from $P_{1}(s) . P_{i}(s)$ is the set of the $i^{\text {th }}$ step reachable states from $s$ via $E_{i} . A_{i}(v), v \in V_{i}$, is the set of states which will be limited by the $(i+1)^{t h}$ step edges $E_{i+1}$. For $s \in A_{i}(v)$, $\operatorname{ban}(v)$ will be removed from $P_{i}(s)$. We continue our computation $P_{i}(s)$ until no states are removable from $P_{i}(s)$. As a result, $P_{i}(s)$ monotonically decreases to a fix-point $P^{*}(s)$. Thus, we obtain a model $M_{\_} r, p o s t \_M \_r(s)=P^{*}(s)$.

\section{Algorithm: CSM(M,G)}

1. $\forall s \in S, P_{1}(s)=$ post_$_{-} M(s), Q_{1}\left(v_{0}\right)=S$,

2. For $i$ from 1 to $t-1$ do

3. for $v \in V_{i}$,

4. $\quad A_{i}(v)=\cup_{e \in E_{i} \cap \operatorname{end}(v)}\left[\operatorname{ant}(e) \cap Q_{i}(\operatorname{start}(e))\right]$;

5. $\quad \operatorname{ban}(v)=\cup_{e \in \operatorname{start}(v)} \operatorname{ban}(e)$;

6. If $s \in A_{i}(v)$, then $P_{i+1}(s)=P_{i}(s)-\operatorname{ban}(v)$;

7. Else, $P_{i+1}(s)=P_{i}(s)$;

8. $\quad Q_{i+1}(v)=\cup_{s \in A_{i}(v)} P_{i+1}(s)$;

9. End For.

10. $k=t$;

11. For $j$ from 1 to $\tau-1$ do

12. $i=k-1+j$;

13. for $v \in V_{i}$,

14. $A_{i}(v)=\cup_{e \in E_{i} \cap \operatorname{end}(v)}\left[\operatorname{ant}(e) \cap Q_{i}(\operatorname{start}(e))\right]$;

15. $\quad \operatorname{ban}(v)=\cup_{e \in \operatorname{start}(v)} \operatorname{ban}(e)$;

16. If $s \in A_{i}(v)$, then $P_{i+1}(s)=P_{i}(s)-\operatorname{ban}(v)$;

17. Else, $P_{i+1}(s)=P_{i}(s)$;

18. $\quad Q_{i+1}(v)=\cup_{s \in A_{i}(v)} P_{i+1}(s)$;

19. End For.

20. If $P_{k+\tau}(s)=P_{k}(s)$ for all $s \in S$ and $Q_{k+\tau}(v)=Q_{k}(v)$, for $v \in V_{k}$, goto 22 ;

21. Else $k=k+\tau$; goto 11 ;

22. Return a model $M \_r$, where post_M_r $(s)=P^{*}(s)=P_{k}(s)$ for all $s \in S$.

Lemma 2. The algorithm CSM stops in a finite number of steps.

Theorem 5. $M \_r \models G$.

We use CSM to find a maximal model of $G$. We start from a trivial model $M_{0}:$ post_ $M_{0}(s)=S$ for all $s \in S$. Using CSM, we get a satisfying model $M_{\_} r=C S M\left(M_{0}\right)$. But in some cases, $M_{\_} r$ may not be a maximal model. For instance, when we calculate $A_{2}(v)$ to do the third edge's limitation, $A_{2}(v)=$ $\cup_{e \in E_{2} \cap e n d(v)}\left[\operatorname{ant}(e) \cap \cup_{s \in A_{1}(\operatorname{start}(e))} P_{2}(s)\right]$. Because $P_{2}(s) \supseteq P^{*}(s)$, it is possible that $A_{2}(v) \supset \cup_{e \in E_{2} \cap e n d(v)}\left[\operatorname{ant}(e) \cap \cup_{s \in A_{1}(\operatorname{start}(e))} P_{2}(s)\right]$. As a result, there are more states which are taken off from the set of reachable states set during the third step. To avoid this, we have to start from a refined model $M$ which is smaller than $M_{0}$ but no more than a maximal model. The following algorithm, called Induced Model $(I M)$, is used to find such an initial model. 


\section{Algorithm: IM(M,G)}

1. $\forall s \in S, P_{1}(s)=S, R_{1}\left(v_{0}\right)=S$,

2. For $i$ from 1 to $t-1$ do

3. for $v \in V_{i}$,

4. $\quad B_{i}(v)=\cup_{e \in E_{i} \cap e n d(v)}\left[\operatorname{ant}(e) \cap R_{i}(\operatorname{start}(e))\right]$;

5. $\quad \operatorname{ban}(v)=\cup_{e \in \operatorname{start}(v)} \operatorname{ban}(e)$;

6. If $s \in B_{i}(v)$, then $P_{i+1}(s)=P_{i}(s)-\operatorname{ban}(v)$;

7. $\quad$ Else, $P_{i+1}(s)=P_{i}(s)$;

8. $\quad R_{i+1}(v)=\cup_{s \in B_{i}(v)}$ post_M(s);

9. End For.

10. $k=t$;

11. For $j$ from 1 to $\tau-1$ do

12. $i=k-1+j$;

13. for $v \in V_{i}$,

14. $\quad B_{i}(v)=\cup_{e \in E_{i} \cap \operatorname{end}(v)}\left[\operatorname{ant}(e) \cap R_{i}(\operatorname{start}(e))\right]$;

15. $\quad \operatorname{ban}(v)=\cup_{e \in \operatorname{start}(v)} \operatorname{ban}(e)$;

16. If $s \in B_{i}(v)$, then $P_{i+1}(s)=P_{i}(s)-\operatorname{ban}(v)$;

17. $\quad$ Else, $P_{i+1}(s)=P_{i}(s)$;

18. $R_{i+1}(v)=\cup_{s \in A_{i}(v)}$ post_M(s);

19. End For.

20. If $R_{k+\tau}(v)=R_{k}(v)$, for $v \in V_{k}$, goto 22 ;

21. Else $k=k+\tau$; goto 11;

22. Return a model $M_{-} r$, where post_M_ $M_{-}(s)=P^{*}(s)=P_{k}(s)$ for all $s \in S$.

Theorem 6. If $M \models G$, then the output model in $\operatorname{IMIM}(M, G) \geq M$.

Theorem 7. If $M_{1} \geq M_{2}$, and $M_{1} \models G$, then $\operatorname{IM}\left(M_{2}, G\right) \geq \operatorname{IM}\left(M_{1}, G\right) \geq$ $M_{1} \geq M_{2}$.

Theorem 8. If $M \models G$ and $I M(M, G)=M$, then $M$ is a maximal model of $G$.

\section{Algorithm: $\operatorname{RCSM}(\mathbf{G})$}

1. $F \operatorname{lag} 1=F \operatorname{lag} 2=0 ; k=1$;

2. $M_{-} r[1]=C S M\left(M_{0}, G\right)$;

3. While Flag $1=0$ do

4. $M_{k}=I M\left(M_{-} r[k], G\right)$;

5. $\quad M_{-} r[k+1]=C R M\left(M_{k}, G\right)$;

6. If $M_{-} r[k+1]>M_{-} r[k]$, then $k=k+1$;

7. $\quad$ Else Flag $1=1$; If $M_{k}=M_{-} r[k]$, then Flag $2=1$;

8. Return: $M \_r r=M \_r[k]$, Flag2 .

Theorem 9. (1) Algorithm RCSM will stop in finite steps.

(2) $M \_r r \models G$.

(3) If Flag2 $=1$, then $M \_r r$ is a maximal model of $G$.

It is still open if $M_{-} r[k]$ monotonically increases. Therefore we cannot guarantee $M_{r} r$ be a maximal model of $G$. But, anyway, even $M_{-} r r$ is not a maximal 


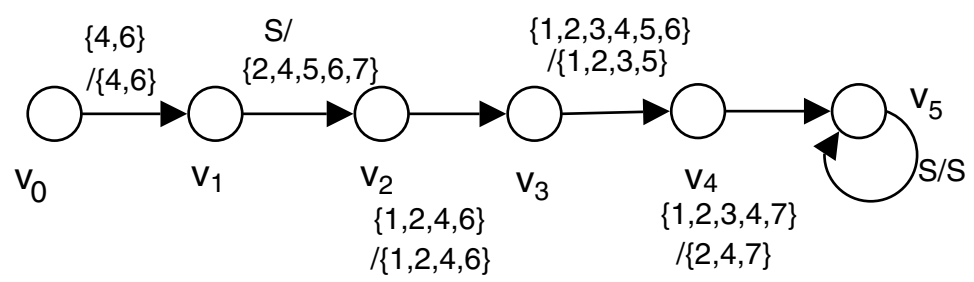

Fig. 4. An assertion graph

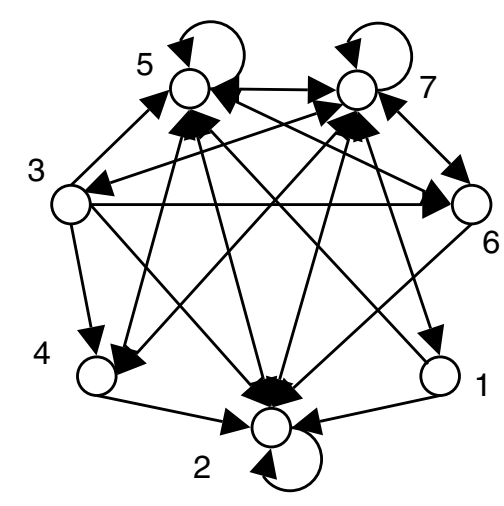

Fig. 5. Model $M_{\_} r[1]$

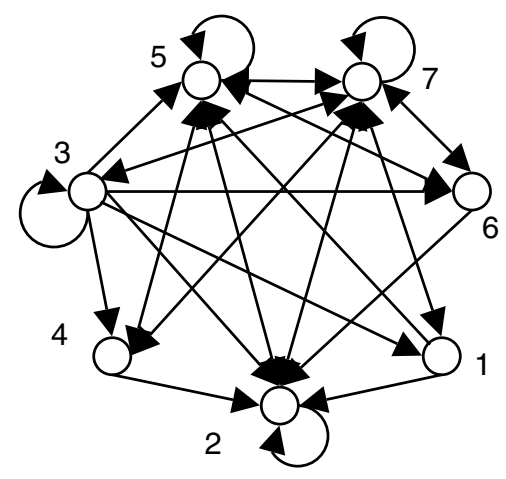

Fig.5(b). Model $M \_r r=M \_r[2]$

model, there is a maximal model $M$ between $M \_r r$ and $I M\left(M \_r r, G\right)$ according to Theorem 7 . The following example shows an application of RCSM to find a maximal model of an assertion graph $G$.

Example 3. Given an assertion graph $G$ as Fig.4.

According to the algorithm RCRM, we get $M_{\_} r[1]$ (Fig.5) as:

$$
\operatorname{Post}(1)=\{2,5,7\}, \operatorname{Post}(2)=\{2,5,7\}, \operatorname{Post}(3)=\{2,4,5,6,7\}, \operatorname{Post}(4)=
$$
$\{2,5,7\}, \operatorname{Post}(5)=\{2,4,5,6,7\}, \operatorname{Post}(6)=\{2,5,7\}, \operatorname{Post}(7)=\{1,2,3,4,5,6,7\}$.

M_r [2] (Fig.5(b)) is: Post (1) $=\{2,5,7\}, \operatorname{Post}(2)=\{2,5,7\}, \operatorname{Post}(3)=$ $\{1,2,3,4,5,6,7\}, \operatorname{Post}(4)=\{2,5,7\}, \operatorname{Post}(5)=\{2,4,5,6,7\}, \operatorname{Post}(6)=\{2,5,7\}$, $\operatorname{Post}(7)=\{1,2,3,4,5,6,7\} . M_{-} r[3]=M_{-}[2]$, so stop. $M_{2}=I M\left(M_{-} r[2], G\right)=$ $M \_r[2]$, so Flag $2=1$, thus $M \_r r=M \_r[2]$ is a maximal model of $G$ (Theorem 5.4). $M_{\_} r[2]>M_{\_} r[1]$ (There are two more edges: $(3,3),(3,1)$ in $M_{\_} r[2]$ than in $\left.M_{\_} r[1]\right)$.

\section{Conclusions}

We presented a new model characterization called maximal models for an assertion graph with important properties. We showed that an assertion graph may have more than one maximal model. We presented a provable algorithm to find all maximal models of a linear assertion graph. We devised an algorithm for finding a maximal model for an arbitrary assertion graph. 


\section{References}

[1] Yang, J. and Seger, C.: Generalized Symbolic Trajectory Evaluation. Technical Report, 2002, Intel.

[2] Yang, J. and Seger, C.: Introduction to Generalized Symbolic Trajectory Evaluation. IEEE Trans on VLSI Systems, 11(3) (2003), 345-353

[3] Hu, A. J., Casas, J., and Yang, J.: Efficient Generation of Monitor Circuits for GSTE Assertion Graphs. IEEE/ACM International Conference on ComputerAided Design (2003), 154-159

[4] Hu, A. J., Casas, J., and Yang, J.: Reasoning about GSTE Assertion Graphs. 12th Ad-vanced Research Working Conference on Correct Hardware Design and Verification Methods (CHARME) (2003), 170-184

[5] Seger, C. and Bryant, R. E.: Formal verification by symbolic evaluation of partially-ordered trajectories. Formal Methods in System Design, 6(2) (1995): 147-190

[6] Yang, G. , Yang, J. and Song, X.: Formal Verification by Generalized Symbolic Trajectory Evaluation. Technical Report, Portland State University, 2004

[7] Chou, C.-T.: The mathematical foundation of synmbolic trajectory evaluation, in Proc. Computer-aided Verifucation(CAV) (1999), 196-207

[8] Bentley, B.: High level validation of next generation microprocessors. Proc. IEEE High Level Design Validation and Test Workshop (HLDVT) (2002), 31-35

[9] Aagaard, M., Jones, R. B., and Seger, C.: Combining thereom proving and trajectoy evaluaton in an industrial environment, in 35th Design Automation Conferece, ACM/IEEE (1998), 538-541

[10] Bjesse, P., Leonard, T., and Mokkedem, A.: Finding bugs in an Alpha microprocessor using satisfiability solvers, in Computer-Aided Verification: 13th International Conferece, pages 454-464

[11] Kyle L. Nelson Alok Jain, and Bryant, R. E.: Formal verification of a superscalar execution unit, in 34th Design Automation Conferece, ACM/IEEE (1997), 161167

[12] Pandey, M., Raimi, R., Beatty, D. L., and Bryant, R. E.: Formal verification of PowerPC arrays using symbolic trajectory evluation, in 33rd Design Automation Confer-ence, ACM/IEEE (1996), 649-654

[13] Edmund M. Clarke and E. Allen Emerson: Design and synthesis of synchronization skele-tons using branching time temporal logic, in Dexter Kozen, editor, Workshop, on Logics of Programs (May 1981), 52-71

[14] Kurshan, R. P.: Computer-Aided Verification of Coordinating Processes: The automata-Theorotic Approach, Princeton University Press, 1994

[15] Yang, G., Yang, J. Hung, W.N.N, and Song, X.: Implication of assertion graphs in GSTE, ASPDAC (2005), 1060-1063 